\title{
Beyond research communication...
}

Sri Lanka's progress towards middle income status is greatly dependent on correct development policies and programmes based on sound scientific evidence. This requires the generation of knowledge through well designed and ethically sound research. There is a large gap between knowledge generation and its utilisation the world over. The growing body of literature on how to utilise research findings for economic gains shows that a substantial proportion of research output remains unutilised. Finding ways to improve the application of research results remains a formidable challenge. In this context it is important to identify the barriers that prevent the utilisation of research findings and to develop strategies to overcome these.

Research is not a luxury to be enjoyed only by scientists in countries with plenty of resources. In the words of Jawaharlal Nehru, "because we are a poor country, we cannot afford not to do research" ${ }^{1}$. In addressing national problems, local scientists have a major role to play, especially in operational research. This is best prioritized, designed and implemented within a national framework. In this respect, it is necessary to prioritize the improvement of the capacity of national research institutions and invest in development of human capital, by nurturing a pool of scientists who think creatively, are capable of innovation and have the required technological expertise.

Scientists often prefer the academic freedom to pursue scientific curiosity. However, compromises are made to conduct research in an area where funding is available. The rising cost of research and the competitive demands for "donor" funding has led to a shift in emphasis towards applied research especially in developing country settings. However, if research is to be directed towards addressing development problems of national importance, then it becomes crucial that funds be made available for such work without dependency on "outside" funds. This means substantial investments in the research process recognising it as a pre requisite for national development.
The above alone however, is insufficient to improve the relevance and utilisation of findings. For this to happen there should be mechanisms for close and continuing dialogue between researchers, policy makers and programme implementers. The lack of such dialogue is identified as one of the key obstacles to utilisation of research. For this process to work effectively policy makers have to appreciate the contribution that research can make towards development on the one hand and on the other, researchers must have skills necessary to utilize a range of dissemination strategies to suit the differing needs of stakeholders. Although the need for rigorous training in research methodology and technology is identified as essential to generate good research, the need to improve a researcher's skills in communication beyond a scientific paper or presentation is not often recognised.

Scientific communication as a paper or as a presentation at a scientific meeting serves the purpose of exposing the work to scientific review by peers and serves to establish the scientific validity of results. Such communications rarely reach policy makers, planners and implementers. The findings have to be packaged differently if this group is to be reached and may need to take the form of a policy brief or a document for advocacy. Findings that are of importance to the general public have to be presented in an easily understood form, utilising methods that reach the majority of the target population. The dissemination needs other than scientific communications are not often addressed in research budgets and are therefore not met.

The model that is currently used to get research into practice is one where the researchers "discover" a solution and then try to "sell" it to the decision makers. However, selecting an "identified priority need" as an area for research provides a good opportunity to establish a dialogue with potential users from the inception of the research process. Often, converting research into practice requires a "scaling-up" of activities. Alliances between researchers and stakeholders will enable researchers to examine the problem from the perspective of the decision

1. Fathalla M.F. \& Fathalla M.M.F. (2004). A Practical Guide for Health Researchers p.11. WHO Regional Office for the Eastern Mediterranean, Cairo. 
makers and implementers, think about the practicality of scaling up of research findings, planning actions required at operational level and to address some of the macro contextual barriers to implementation. Such links also provide opportunities for researchers to act as catalysts for action within national programmes. Research findings are more likely to be considered if the credibility of the research teams and institutions are well established. Links between researchers and policy makers enable the later to recognise and appreciate the capabilities of the former.

Collaboration between research groups addressing the different aspects of a problem will optimise utilisation of scarce resources, add weight to advocacy and enhance the likelihood of utilisation of findings. Currently, most such collaborations are researcher dependent and ad hoc. Mechanisms that will facilitate such collaboration need to be planned and institutionalised.
It is important to address the question of, whether it is the responsibility of researchers to ensure utilisation of their research findings. Is it fair to expect them to don the many hats required for this function? Would utilisation be better served by a group of experts whose primary function would be to acquire, analyse and synthesise information for use by policy makers? Such a "think tank" could also serve to identify gaps in knowledge, suggest areas for further research, prioritise competing demands, facilitate collaboration between researchers and help procure funding, forming a two way bridge between researchers and policy makers. Irrespective of the mechanism, the priority need in our country, is to institutionalise a culture of evidence based decision making within the national planning process. "Because we are a poor country", not only can we "not afford not do research", we cannot also afford not to make use of research findings.

Lalini Rajapaksa 